\title{
Pulmonary Sclerosing Pneumocytoma and Adenocarcinoma Presenting as Two Distinct Contralateral Neoplasms in the Same Patient
}

\author{
Oliveira $T^{1,2}$, Shekhovtsova $\mathbf{M}^{3}$, Vicente $\mathbf{P}^{1}$, \\ Miranda J ${ }^{4}$, Araújo $A^{2,5 *}$ and Guedes $F^{1,2}$ \\ ${ }^{1}$ Department Pulmonology, Centro Hospitalar \\ Universitário do Porto, Portugal \\ 2Instituto de Ciências Biomédicas Abel Salazar, \\ Universidade do Porto, Portugal \\ ${ }^{3}$ Department of Anatomic Pathology, Centro Hospitalar \\ Vila Nova de Gaia/ Espinho, Portugal \\ ${ }^{4}$ Department of Cardiothoracic Surgery, Centro \\ Hospitalar Vila Nova de Gaia/ Espinho, Portugal \\ ${ }^{5}$ Department of Medical Oncology, Centro Hospitalar \\ Universitário do Porto, Portugal \\ *Corresponding author: Araújo A, Medical Oncology \\ Department, Centro Hospitalar Universitário do Porto, \\ Hospital de Santo António, Porto, Portugal
}

Received: January 28, 2019; Accepted: March 01, 2019; Published: March 08, 2019

\section{Abbreviations}

AJCC: American Joint Committee on Cancer; B7: Medial Segment of the Right Lower Lung Lobe; CT: Computed Tomography; CK: Cytokeratin; DLCO: Diffusion Capacity of the Lung for Carbon Monoxide; ECOG-PS: Eastern Cooperative Oncology GroupPerformance Status; EGFR: Epidermal Growth Factor Receptor; ${ }^{18}$ F-FDG: Fluorodeoxyglucose; KCO: Krogh Index (diffusion capacity of the lung for carbon monoxide corrected for alveolar volume); PD-L1: Programmed Death-Ligand 1; PET-CT: Positron Emission Tomography-Computed Tomography; SUV(max): maximum Standardized Uptake Value; TTF-1: Thyroid Transcription Factor-1

\section{Case Presentation}

A 65-year-old non-smoker woman, with occasional exposure to second-hand smoke and no relevant occupational inhalation exposure, ECOG-PS 0, presents to her family physician with a threemonth history of progressive exertional dyspnea and productive cough with occasional hemoptoic expectoration. Constitutional symptoms or other organ-specific symptoms were absent. Chronic gastritis, hypercholesterolemia, non-insulin-treated diabetes mellitus, hypertension and osteoarthrosis were her major comorbidities. Personal or familial history of respiratory or neoplastic diseases was absent.

Empiric antibiotic therapy, inhaled corticosteroid and longacting bronchodilators were prescribed, leading to transient clinical improvement. The patient was referred to Pneumology outpatient consultation for further urgent investigation.

Postero-anterior and left lateral chest radiographs were unremarkable. However, thoracic CT scan showed both a solid oval

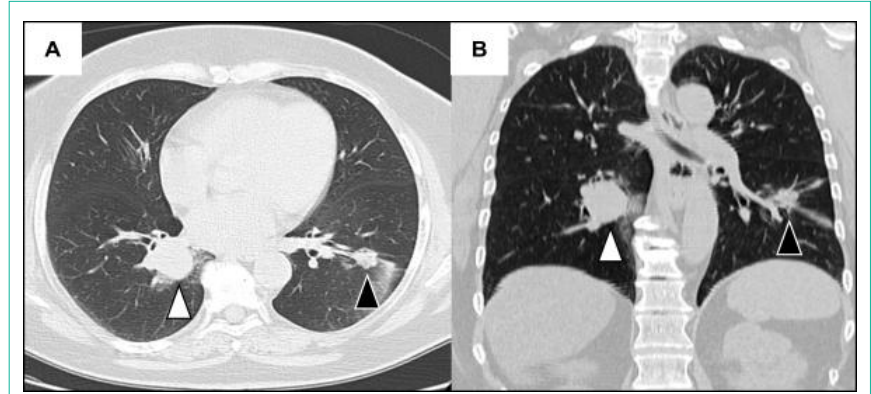

Figure 1: Thoracic CT scan images (parenchymal window) showing the two contralateral lung nodules: on the right lung, white arrow with black outline: $28.4 \times 25.5 \mathrm{~mm}$ pulmonary sclerosing pneumocytoma of the right lower lobe; on the left lung, black arrow with white outline: $14 \times 16 \mathrm{~mm}$ lung adenocarcinoma. (A) Axial plane. (B) Coronal plane.

regular $27 \mathrm{~mm}$ right infra-hilar lung nodule and a heterogeneous 15 $\mathrm{mm}$ left hilar lung nodule (Figure 1). The remaining imaging of lung parenchyma was normal and mediastinal or pleural involvement were absent.

Fiberoptic bronchoscopy showed enlargement of B7 division and reduction of $\mathrm{B} 7$ bronchus caliber; there were no other relevant endoscopic findings. Bronchial biopsy and bronchial wash were negative for neoplastic cells. No microbiological agents were isolated in the bronchial aspirate.

Dual-phase whole-body ${ }^{18} \mathrm{~F}-\mathrm{FDG}$ PET-CT scan showed strong ${ }^{18} \mathrm{~F}-\mathrm{FDG}$ uptake in both lung nodules, suspicious of malignancy: SUV (max) in the delayed scan was equal to 3.9 in the right nodule and 4.4 in the left nodule (Figure 2). Significantly increased ${ }^{18} \mathrm{~F}-\mathrm{FDG}$ uptake was absent elsewhere.
Ann Hematol Oncol - Volume 6 Issue 4 - 2019

ISSN : 2375-7965 | www.austinpublishing group.com

Araújo et al. (C) All rights are reserved
Citation: Oliveira T, Shekhovtsova M, Vicente P, Miranda J, Araújo A and Guedes F. Pulmonary Sclerosing Pneumocytoma and Adenocarcinoma Presenting as Two Distinct Contralateral Neoplasms in the Same Patient. Ann Hematol Oncol. 2019; 6(4): 1242. 
A

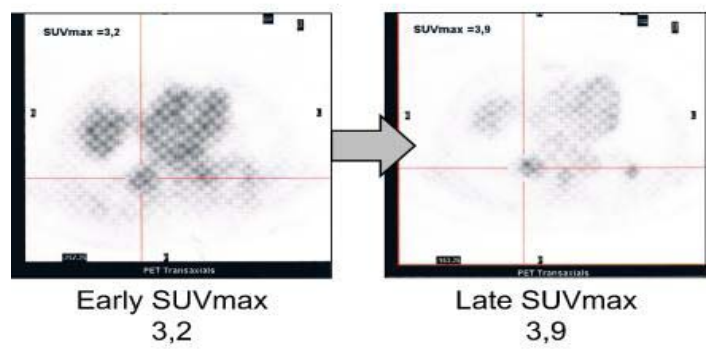

B

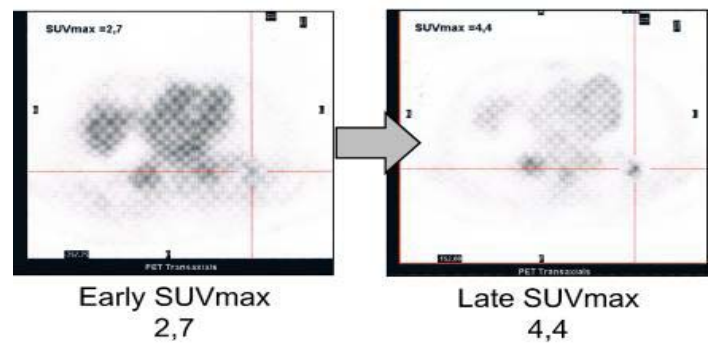

Figure 2: Dual phase ${ }^{18}$ F-FDG PET-CT scan images of both lung nodules: the early scans are shown on the left and the delayed scans are shown on the right. (A) Right lung nodule (pulmonary sclerosing pneumocytoma). (B) Left lung nodule (lung adenocarcinoma).

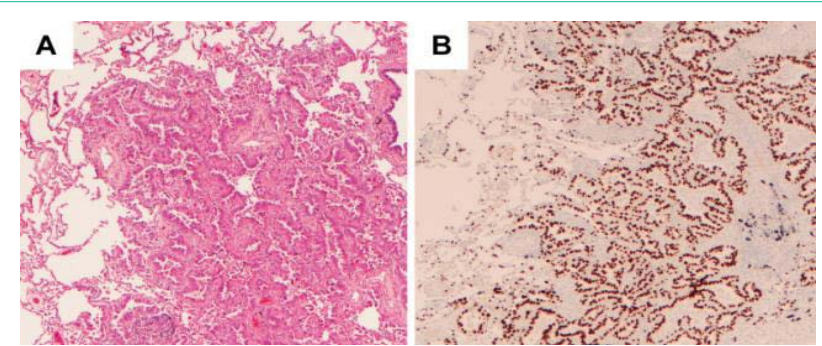

Figure 3: Histologic features of lung adenocarcinoma. (A) The tumor was well-differentiated and presented a predominantly acinar and lepidic pattern (hematoxylin and eosin stain, 20x magnification). (B) Immunostaining showing strong positivity of tumor cells for TTF1.

Biopsy of both lung nodules was performed. Firstly, transthoracic needle biopsy of the right lung nodule revealed a pulmonary sclerosing pneumocytoma. Then, transthoracic needle biopsy of the left lung nodule led to the diagnosis of lung adenocarcinoma; EGFR mutation was present and PD-L1 expression was absent.

Lung volumes measured by body plethysmography were normal and both DLCO and KCO (single breath method) were slightly decreased.

Lower left lung lobe uniportal video-assisted thoracoscopic lobectomy was performed for adenocarcinoma removal with no complications. The histology of the surgical specimen demonstrated well differentiated lung adenocarcinoma with predominant acinar and lepidic pattern and there were no ganglionic metastases (corresponding to pT2aN0 stage, according to AJCC Cancer Staging Manual, eighth edition) (Figure 3). Perineural permeation
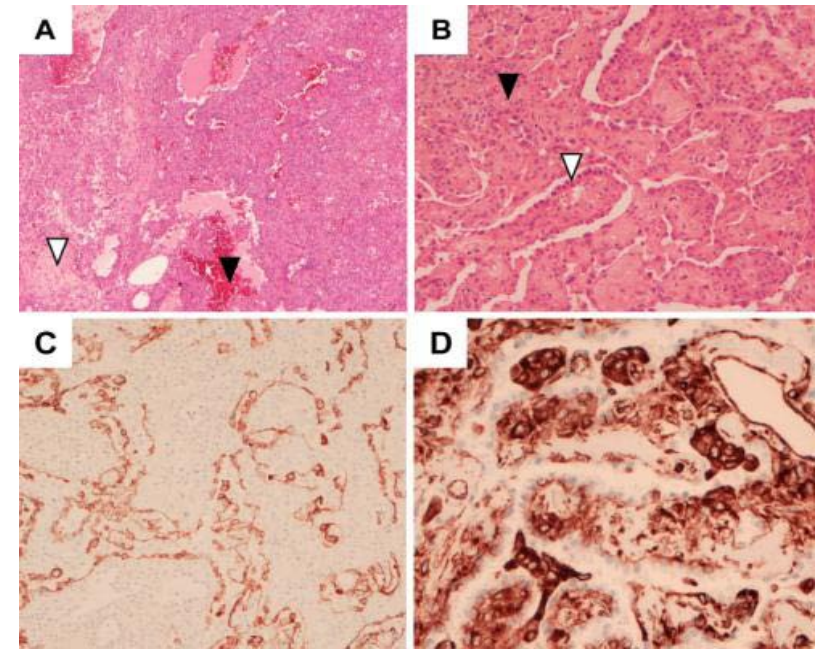

Figure 4: Histologic features of pulmonary sclerosing pneumocytoma. (A) Note the sclerotic (white arrowhead) and hemorrhagic (black arrowhead) regions (hematoxylin and eosin stain, 20x magnification). (B) Cuboida surface cells (white arrowhead with black outline) and round stromal cells (black arrowhead) can be identified in the tumor (hematoxylin and eosin stain, 200x magnification). (C) CK7 positivity in cuboidal surface cells. (D) Vimentin positivity in round stromal cells.

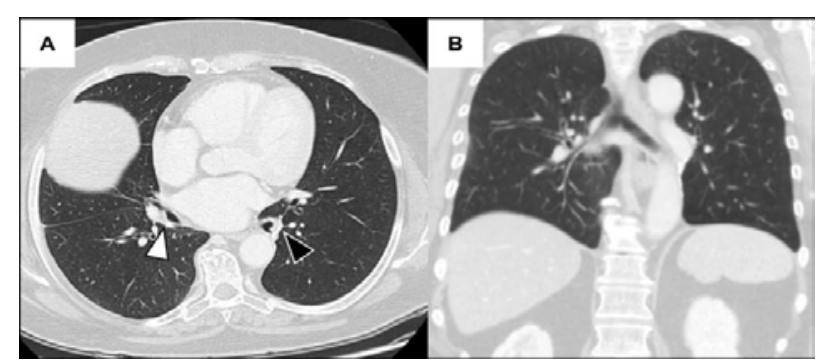

Figure 5: Thoracic CT scan images (parenchymal window) acquired in the follow-up period after left and right lobectomies. (A) Axial plane (roughly at the same anatomic level of figure $1 \mathrm{~A}$ ). On the right lung, white arrow with black outline: bronchial stump of the right lower lobe bronchus; on the left lung, black arrow with white outline: bronchial stump of the left lower lobe bronchus. Note the fibro-cicatricial thickening of both bronchial stumps. (B) Coronal plane (roughly at the same anatomic level of figure $1 \mathrm{~B}$ ).

and lymphovascular invasion were identified. Thus, adjuvant chemotherapy with cisplatin and vinorelbine was started and welltolerated by the patient.

Thoracic CT scan after chemotherapy revealed stability of the pulmonary sclerosing pneumocytoma and absence of new relevant lesions.

Lower right lung lobe uniportal video-assisted thoracoscopic lobectomy was subsequently performed for pulmonary sclerosing pneumocytoma removal with no complications. The histology of the surgical specimen confirmed the initial diagnosis and showed tumor with predominant papillary and, focally, acinar or solid pattern, with sclerotic and hemorrhagic regions. This lesion was composed by two types of neoplastic cells-cuboidal surface cells and stromal round cells with slightly different immunohistochemical profiles. Surface cells were positive for TTF1, CK7 and pan cytokeratin AE1/AE3 and negative for vimentin. On the other hand, stromal cells were positive 
for TTF1 and vimentine, but negative for CK7 and AE1/AE3 (Figure 4). Neither perineural or lymphovascular invasion nor ganglionic involvement were found.

Nine months after surgery, the patient remains completely asymptomatic and free of lung neoplastic disease. Follow-up thoracic CT scans revealed fibro-cicatricial changes in the subpleural, anterolateral aspect of the middle lung lobe and in both bronchial stumps; these imaging studies were otherwise unremarkable (Figure $5)$.

\section{Discussion}

This patient was sequentially diagnosed two distinct contralateral lung neoplasms. Both lesions had a ${ }^{18} \mathrm{~F}$-FDG uptake in ${ }^{18} \mathrm{~F}$-FDG PETCT scan highly suggestive of malignancy, however the right lung lesion was benign, and the left lung lesion was malignant. Pulmonary sclerosing pneumocytoma is a rare, benign lung neoplasm, normally occurring as a solitary tumor, with a predilection for middle-aged women and is a mimicker of lung cancer because of similar ${ }^{18} \mathrm{~F}-\mathrm{FDG}$ uptake; clinical course is typically benign and metastization is very rare [1-3].

This clinical case raises questions regarding lung biopsy decisions in patients with multiple lung nodules or masses with similarly strong ${ }^{18} \mathrm{~F}$-FDG uptake-those lung lesions may be separate neoplastic diseases with distinct clinical behaviors, which might have significant implications on staging and therapeutic decisions.

\section{References}

1. Shin SY, Kim MY, Oh SY, Lee HJ, Hong SA, Jang SJ, Kim SS, et al. Pulmonary Sclerosing Pneumocytoma of the Lung: CT Characteristics in a Large Series of a Tertiary Referral Center. Medicine. 2015; 94: e498.

2. Lim JH, Lee $\mathrm{N}$, Dae Woong $\mathrm{C}$, Hyung Joo O, Park $\mathrm{YH}$, Kim KH, et al. Pulmonary sclerosing pneumocytoma mimicking lung cancer: Case report and review of the literature. Thoracic Cancer. 2016; 7: 508-511.

3. Kalhor N, Staerkel GA, Moran CA. So-called sclerosing hemangioma of lung: current concept. Ann Diagn Pathol. 2010; 14: 60-67.
Ann Hematol Oncol - Volume 6 Issue 4 - 2019

ISSN : 2375-7965 | www.austinpublishinggroup.com Araújo et al. (C) All rights are reserved
Citation: Oliveira T, Shekhovtsova M, Vicente P, Miranda J, Araújo A and Guedes F. Pulmonary Sclerosing Pneumocytoma and Adenocarcinoma Presenting as Two Distinct Contralateral Neoplasms in the Same Patient. Ann Hematol Oncol. 2019; 6(4): 1242. 